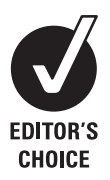

Clinical Motor Function Laboratory, The Parker Institute, Frederiksberg Hospital, Frederiksberg, Denmark ${ }^{2}$ Department of Neuroscience and Pharmacology, Division of Biomechanics, The Panum Institute, University of Copenhagen, Copenhagen, Denmark

Correspondence to: Marius Henriksen, Clinical Motor Function Laboratory, The Parker Institute, Frederiksberg Hospital, Ndr. Fasanvej 57, DK-2000 Frederiksberg, Denmark; marius. henriksen@frh.regionh.dk

Accepted 11 August 2008 Published Online First 21 August 2008

\title{
Experimental muscle pain during a forward lunge the effects on knee joint dynamics and electromyographic activity
}

\author{
M Henriksen, ${ }^{\top}$ T Alkjær, ${ }^{2}$ E B Simonsen, ${ }^{2}$ H B Biddal'
}

\section{ABSTRACT}

Objective: The purpose of this study was to investigate whether the knee joint dynamics during a forward lunge could be modulated by experimentally induced vastus medialis pain in healthy subjects.

Design: Randomised cross-over study.

Setting: Biomechanical movement laboratory.

Participants: 20 healthy subjects were included. One subject was excluded during data collection.

Intervention: The subjects performed forward lunges before, during and 20 minutes after induction of experimental quadriceps muscle pain. Muscle pain was induced using hypertonic saline (5.8\%) injected intramuscularly. Isotonic saline $(0.9 \%)$ was used as control.

Main outcome measurements: Three-dimensional movement analyses were performed and inverse dynamics were used to calculate joint kinematics and kinetics for ankle, knee and hip joints. Electromyographic (EMG) signals of the hamstrings and quadriceps muscles were recorded.

Results: During and after pain, significant decreases in knee joint dynamics and EMG recordings were observed. Conclusion: The study shows that local pain in the quadriceps is capable of modulating movements with high knee joint dynamics. The results may have implications in the management of muscle pain and prevention of injuries during activities involving the knee joint.

Knee pain is among the most common musculoskeletal pain complaints, ${ }^{1}$ and is the most frequent reason for reduced physical function. ${ }^{2}$ Furthermore, knee pain is the cardinal symptom in many diseases and disorders of the knee, such as osteoarthritis, ${ }^{3}{ }^{4}$ tendonitis ${ }^{5}$ and traumatic knee injuries. ${ }^{6}$ Because good knee joint function is important for personal independence, ${ }^{7}$ knee pain poses a serious threat to everyday activities such as walking, which is a determinant of overall functional level. ${ }^{8}$ We have previously shown that experimental vastus medialis pain modulates the quadriceps function during the stance phase of walking, leading to impaired knee joint control. ${ }^{9}$ The changes were not only observed during pain, but were sustained for at least 20 minutes after pain. The changes caused by experimental muscle pain were similar to those observed in patients with "real" knee pain, ${ }^{10} 11$ knee injuries ${ }^{12}$ and muscle weakness. ${ }^{14}$

Although the quadriceps muscle is important for translational, torsional and rotatory stiffness of the knee, ${ }^{15-19}$ it has been shown that it is possible to walk without knee extensor moment ${ }^{9} 20$ - a walking pattern known as quadriceps avoidance gait. $^{21} 22$ Such adaptations in knee joint dynamics may be due to the fact that walking requires submaximal knee joint dynamics, and other muscle groups than the quadriceps can generate knee joint dynamics necessary for normal walking. ${ }^{20}$ It is therefore conceivable that quadriceps pain can cause muscular deficiency, while the ability to walk is retained.

If a movement puts higher demands on the knee joint dynamics than walking, it is possible that the central nervous system overrules afferent nociceptive signals, in order to complete the movement efficiently and safely. Muscle pain has been shown to reduce maximal voluntary isometric contractions (MVICs) of the knee extensors. ${ }^{23}$ However, while MVICs performed in an isokinetic dynamometer require maximal muscle activations, MVICs challenge knee joint stability to a limited extent. The forward lunge represents a movement that is primarily controlled by the quadriceps, requires relatively high knee joint dynamics and challenges knee joint stability, while having functional and clinical relevance. ${ }^{24}$ If high-dynamic movements become impaired following muscle pain, it may have important clinical implications for both prevention and rehabilitation of knee joint diseases and injuries.

Accordingly, the purpose of this study was to investigate whether the knee joint dynamics during a forward lunge could be modulated by experimentally induced vastus medialis pain in healthy subjects.

\section{MATERIAL AND METHODS \\ Subjects}

Twenty healthy subjects (10 women and 10 men) with no history of lower extremity pathology, trauma or pain were included. The age, body weight, and height of the subjects were 26.3 (SD 5.8) years, $69.9(8.5) \mathrm{kg}$ and $1.75(0.07) \mathrm{m}$, respectively. One female subject did not manage to complete the forward lunge movement within the preset time of data sampling. Thus, this particular subject was excluded from the study sample. All subjects gave their informed consent before inclusion and the study was approved by the local ethics committee (J.no. [KF] 01-258645).

\section{Study design}

The study was designed as a cross-over study with each subject tested on 2 days separated by at least 1 week. During a test day, three series of forward lunge tests were performed. Series 1 and 2 were separated by a 5 minute recess, during which the 
subjects rested in a chair. Between series 2 and 3, a 20 minute break was held. Thus, forward lunge tests were performed at the following time points: 0,5 and 25 minutes. Immediately before series 2 intramuscular saline injections into the vastus medialis muscle were given. The saline was either hypertonic $(5.8 \%)$ or isotonic $(0.9 \%)$ saline. On a test day only one type of saline solution was injected, according to the randomisation procedure. The order of the saline solution injections was allocated using an envelope-based randomisation technique, allowing equal numbers of subjects to start with isotonic and with hypertonic saline.

\section{Experimental muscle pain}

Muscle pain was induced by intramuscular bolus injections of $1 \mathrm{ml}$ sterile hypertonic saline (5.8\%) into the right vastus medialis. The injections were given approximately $5 \mathrm{~cm}$ proximal and $5 \mathrm{~cm}$ medial to the medial corner of the patellar base. Injections of isotonic saline $(0.9 \%)$ were used as control. Manual injections over 10 seconds were accomplished by a $5 \mathrm{ml}$ plastic syringe with a disposable needle $(27 \mathrm{G}, 40 \mathrm{~mm})$ at a depth of $3.5 \mathrm{~cm}$. The pain intensity was scored on a $100 \mathrm{~mm}$ visual analogue scale (VAS), where $0 \mathrm{~mm}$ indicated "no pain" and $100 \mathrm{~mm}$ indicated "worst imaginable pain".

\section{Forward lunge procedure}

The subjects stood in an upright position in front of a force plate (model OR6-5-1000, AMTI, Watertown, Massachusetts, USA). They were instructed to perform a forward lunge onto the force plate, lunge until at least $90^{\circ}$ knee flexion and return to an upright position. The subjects were asked to keep their upper body perpendicular to the ground and leave the contralateral foot in contact with the ground during the entire movement. The forward lunge should be completed within 2 seconds, which was also the preset data sampling period. These instructions and settings defined the criteria of a successful forward lunge. Forward lunge movements that did not meet these criteria were excluded from the analysis. The subjects were allowed to practise the forward lunge for as long as they needed to get familiar with the movement before commencing the data recordings. After being accustomed to the testing procedure, the subjects performed consecutive forward lunges until four successful forward lunge trials were recorded. All subjects had their right leg examined.

\section{Biomechanical analysis}

Fifteen reflecting markers were placed on the lower extremities according to the marker set-up described by Vaughan et al. ${ }^{25}$ Five video cameras (Canon MV 600, digital video) operating at 50 frames per second were used to record the movements. Synchronisation between the video signals and the force plate signals was obtained by an audio signal which was transmitted to all five video cameras. Additionally, the signal triggered the recordings of the force platform (sampled at $1000 \mathrm{~Hz}$ ), which ensured synchrony between the video and force signals. The video sequences were digitised and stored on a computer. The video signals and the force plate signals were synchronised electronically with a custom-built device. The data sampling and synchronisation were initiated when the subjects passed a photocell, which was placed in front of the force plate.

Three-dimensional marker coordinates were reconstructed by direct linear transformation using the Ariel Performance Analysis System (APAS, Ariel Dynamics, San Diego, California, USA).
Prior to the calculations, the marker position data were digitally low-pass filtered by a fourth order zero-lag Butterworth filter with a cut-off frequency of $6 \mathrm{~Hz}$, and the $1000 \mathrm{~Hz}$ force plate signals were resampled at 50 sample points per second.

\section{Calculations}

Three-dimensional internal flexor and extensor joint moments about the ankle, knee and hip joints were calculated using a three-dimensional inverse dynamics approach. ${ }^{25}$ The joint moments were expressed in anatomically based reference systems. The contact time of the forward lunge was defined as the time period when the foot was in contact with the ground and determined from the force platform signal. The contact phases of four forward lunge trials were analysed for each subject. Discrete joint moment variables were calculated as impulse moments rather than peak moments, due to the small changes in moment during the forward lunge, making the distinction of a peak value unreliable. Sagittal plane impulse moments for hip, knee and ankle joints together with frontal plane knee impulse moment were calculated. Discrete joint angles were determined as peak hip and knee joint flexion angles, and peak dorsal flexion angles. Typical time-course patterns of the joint moments and angles from the biomechanical analyses are shown in fig 1.

\section{Electromyography (EMG)}

Surface EMG was recorded from the vastus medialis (VM), vastus lateralis (VL), semitendinosus (ST) and biceps femoris (BF) muscles using a bipolar surface electrode configuration (AMBU Blue-Sensor N-00-S, Ballerup, Denmark) with a $2 \mathrm{~cm}$ inter electrode distance. Before the electrodes were mounted, the skin was carefully shaved and rinsed with pure alcohol. The EMG signals were bandpass filtered (10-1000 Hz), pre amplified (input impedance $1 \mathrm{GOhm}$ ) and sampled at $1000 \mathrm{~Hz}$ using a lightweight $(70 \mathrm{~g})$ wireless EMG system with 12 bit analogueto-digital resolution (MO8, Marq-Medical, Farum, Denmark). After placement of the electrodes a measurement of resting EMG activity was made while the subject was sitting quietly on a chair and subsequently maximal EMG activity was measured during maximal isometric contractions performed in an isokinetic dynamometer (KinCom, Chattex Corp., Chattanooga, USA). The EMG signals and the movement analysis data were time synchronised, using the force platform data and a footswitch placed under the right heel of the subjects. Representative rectified EMG signals are shown in fig 2.

For each trial, the raw EMG signals were digitally high- and low-pass filtered (Butterworth fourth order zero-lag filter, cutoff frequencies $20 \mathrm{~Hz}$ and $450 \mathrm{~Hz}$, respectively), full-wave rectified and low-pass filtered (cut-off $15 \mathrm{~Hz}$ ) to construct linear envelopes. For all muscles, mean amplitudes during the contact phase were calculated from the linear envelopes. For each subject the mean amplitude values were normalised to the average of the Time 0 mean amplitudes and expressed as a percentage of this value.

\section{Statistical analysis}

A longitudinal data model was applied to assess multiple repeated measures on the same subject. To model the covariance structure in the analysis of the cross-over, repeated measures data, a restricted/residual maximum likelihood (REML) model was applied using the MIXED procedure of the SAS system, as it enables multiple covariance structures 
Figure 1 Hip, knee and ankle joint $\mathrm{kg}^{*} 100$ ) from one subject during a forward lunge. Positive angle values indicate hip extension, knee flexion and ankle plantar flexion. Positive sagittal joint moments (solid lines) indicate hip flexor, knee extensor and ankle dorsal flexor moment. Positive knee frontal joint moment (dashed line) indicates abductor moment (external varus). The curves represent an average of four forward lunge trials and are time normalised to the contact phase $(x$-axis: $0 \%=$ first contact, $100 \%=$ foot off). angles (degrees) and net moments (Nm/
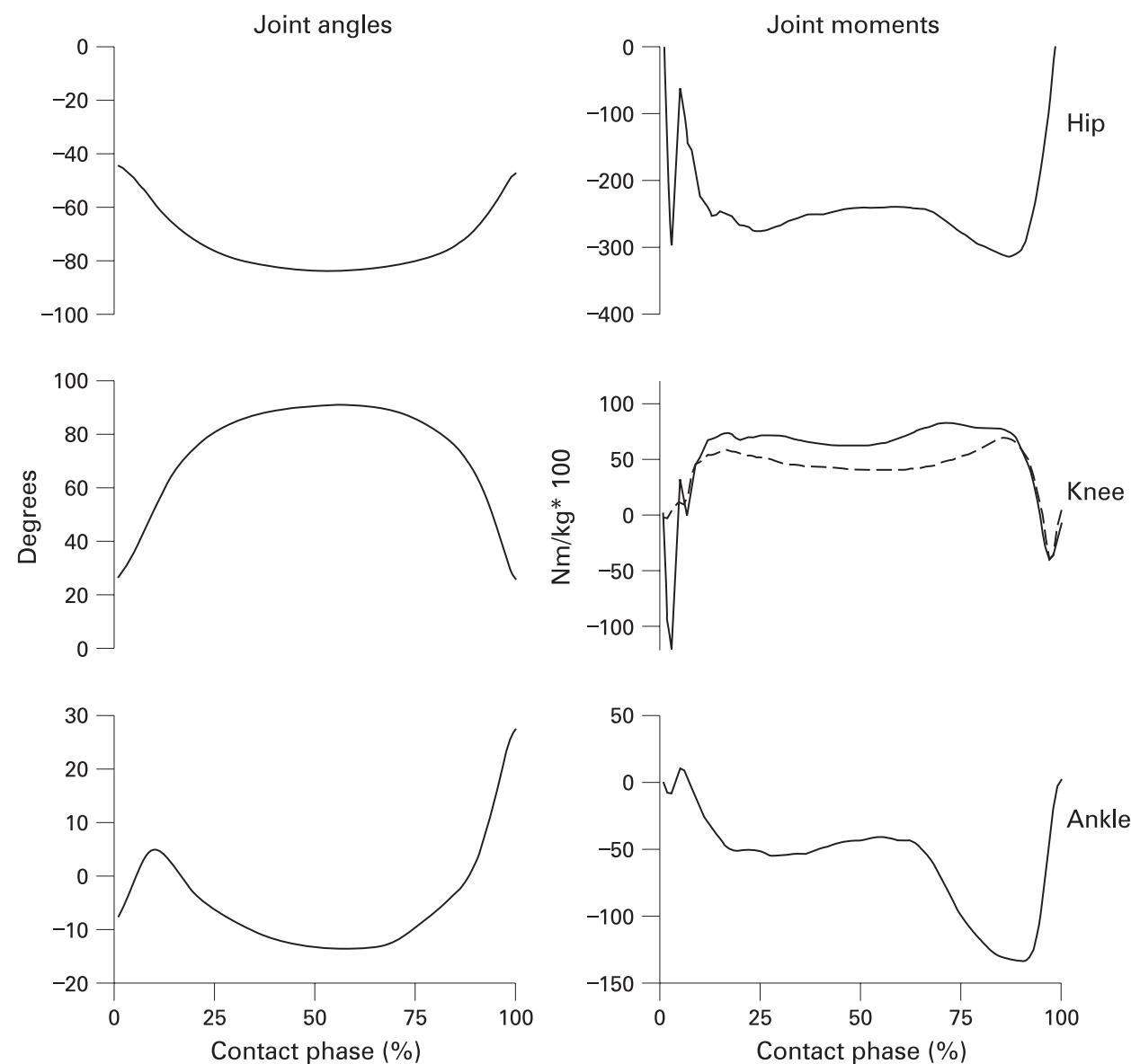

through the RANDOM and REPEATED statements. The analysis focused on the fixed effects analysis, analysing whether there was an interaction: Time $\times$ Saline; applying each factor as main factors in the model, including the corresponding interactions.

Any statistically significant interactions between Saline and Time were broken down post hoc by exploring the pairwise differences of the Saline least-square means at time points 0, 5 and 25 minutes. Statistical significance was accepted at $p<0.05$.

\section{RESULTS}

\section{Muscle pain}

There was a significant interaction between type of Saline and Time $(p<0.001)$. No pain was scored following isotonic saline injections $(p=1)$. Following hypertonic saline injections, the average pain score was $17.3 \mathrm{~mm}$ (95\% confidence interval 15.2 to $19.5 ; \mathrm{p}<0.001)$. There were no pain scores at time 25 minutes $(p=1)$

\section{The forward lunge}

There was no significant Time $\times$ Saline interaction with respect to the contact time $(p=0.383)$.

There were significant Time $\times$ Saline interactions in the impulse of the knee extensor moments $(p<0.001$; see fig 3$)$. Post-hoc analyses showed significantly lower impulse of the knee extensor moments following hypertonic saline at Time 5 $(p<0.001)$ and 25 minutes (impulse moment: $p=0.002)$. There were no significant Time $\times$ Saline interactions in frontal plane impulse moments $(p=0.581)$.
There were no significant Time $\times$ Saline interactions in either hip or ankle dynamics (hip impulse moment: $p=0.344$; ankle impulse moment: $p=0.734$ ).

There was a significant Time $\times$ Saline interaction in knee joint angles $(p=0.044)$, but no significant differences in the pairwise comparison of the least-square means at Time 0,5 and 25 minutes $(p=0.064, p=0.093$, and $p=0.88$, respectively). There were no significant TimexSaline interactions in either hip or ankle kinematics (peak hip angle: $p=0.659$; peak ankle angle: $p=0.357$ )

\section{Electromyography}

There was a significant Time $\times$ Saline interaction in mean amplitudes for all muscles (VM, VL and BF all: $p<0.001$; ST: $p=0.04$ ). Post-hoc analyses showed significantly lower mean amplitudes of all muscles at Time 5 and 25 minutes following hypertonic saline injections, i.e. during and after pain (all: $\mathrm{p}<0.001$; see fig 4).

\section{DISCUSSION}

The main finding of this study was that experimental vastus medialis pain significantly reduces the knee joint dynamics and quadriceps function during a forward lunge, demonstrated as lower knee joint extensor moments and reduced EMG mean amplitudes in the VM and VL muscles. In addition, the EMG amplitudes of the hamstring muscles (BF and ST) were significantly reduced during pain. The decline in both knee joint dynamics and EMG amplitudes was sustained 20 minutes after cessation of pain. The results of the present study corroborate a previous study from our laboratory on the effects of experimental VM pain on knee joint dynamics during walking. ${ }^{9}$ 


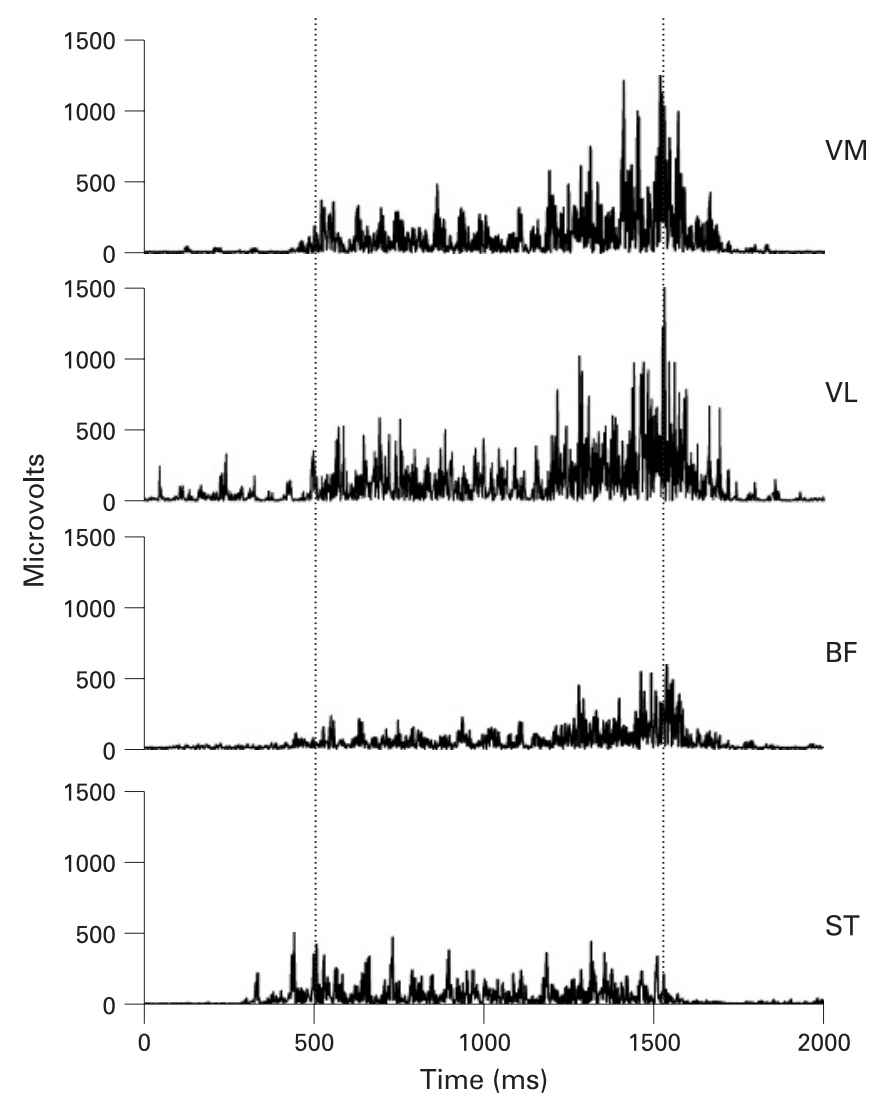

Figure 2 Representative EMG activity of the $m$. vastus lateralis (VL), $m$. vastus medialis (VM), $m$. biceps femoris (BF) and $m$. semitendinosus (ST) from one subject during a forward lunge. The vertical dotted lines indicate the contact phase. The EMG signals, expressed in microvolts, were detrended, high-pass filtered, and rectified.

Muscle pain is mediated by group III and IV afferents ${ }^{26}{ }^{27}$ and has been shown to decrease the excitability of both spinal ${ }^{28-31}$ and cortical motor neuron pools. ${ }^{31}$ These mechanisms may account for the observed changes in muscle activation and movement pattern. The observation of synergist inhibition of $\mathrm{VM}$ and VL in the present study is in accordance with the neuromuscular activation model, ${ }^{32}$ in which pain causes inhibition of synergistic muscles. Inhibition of non-painful synergists has also been reported during isometric contractions of ankle dorsal and plantar flexors, ${ }^{33}$ and it has been shown that experimental muscle pain causes reorganisation of synergistic muscle activity during both dynamic and static contractions. ${ }^{34}{ }^{35}$ The synergistic quadriceps inhibition seen in the present study indicates that central neural control mechanisms are involved in the altered motor activity and movement pattern, as also indicated by a study of experimental muscle pain induced in the rectus femoris muscle, where pain caused reduced maximal voluntary isometric knee extensor torque without impairing the contractile apparatus. ${ }^{23}$

Hamstring muscles (BF and ST) are generally recognised as antagonists to the quadriceps, and reduced hamstring activity was also observed during and after pain. Such reduced hamstring activity reduces the antagonist hamstring moment (knee flexor moment) that the quadriceps must overcome in order to complete the forward lunge with reduced net extensor moment and EMG activity. It has previously been suggested that, during submaximal muscle contractions, the voluntary agonist neural drive can be increased, overcoming any inhibitory mechanisms (e.g. nociceptive), allowing the required muscle

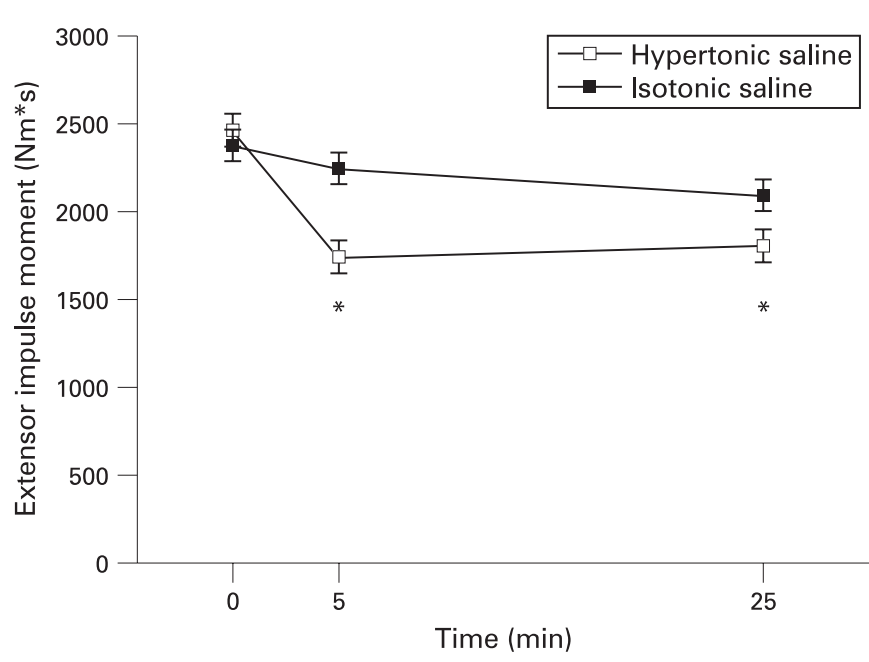

Figure 3 Least-square mean values (SE) of the knee joint extensor impulse moment for effects of isotonic (filled symbols) and hypertonic (open symbols) saline injections into the vastus medialis muscle in 10 male and nine female test subjects. The injections were given immediately before the measurement at Time 5 minutes. There are statistically significant differences (asterisks) between isotonic and hypertonic injections at Time 5 (during pain) and 25 (after pain) minutes.

contractions to be generated. ${ }^{36}$ In contrast, the present results indicate that the nociceptive input causes a reduction of the antagonist muscle activation rather than increasing the agonist neural drive.

The prolonged reduction in joint moment and EMG activity indicates adaptive changes in the movement pattern persisting beyond the conscious pain perception. It is possible that sustained changes following pain may leave the knee joint unstable and prone to injury and may contribute to the chronicity of musculoskeletal problems. The painless synergistic muscle inhibition could indicate a central sensory adaptation to the nociceptive input, ${ }^{37}$ which implies a nociceptive motor modulation at spinal levels with no nociceptive signals reaching supraspinal centres to result in a conscious pain sensation. Another explanation could be that the immediate adaptive response to muscle pain in motor activity and movement pattern is not easily "un-adapted" once the nociceptive input has vanished, but is sustained for at least 20 minutes following pain. The sustained muscle inhibition seen in the present study is in accordance with previous observations showing that inhibition of muscle function by pain still occurs when pain is no longer present. ${ }^{9} 3899$

The knee extensor moment has been shown to have a significant role in stabilising the knee against varus/valgus deformations, ${ }^{15}$ and therefore it follows that reduced extensor moments as observed in the present study may result in knee instability, with medial or lateral opening of the joint, with injuries as the potentially ultimate consequence. As such, the results of this study corroborate the concept of impaired neuromuscular control of the quadriceps as an important risk factor in knee joint injuries. ${ }^{40}$

In conclusion, the present study demonstrates that pain is a powerful sensation with the capability to alter knee joint and quadriceps function even during high-dynamic movements that challenge joint stability. Hence, the notion that the central nervous system discounts afferent nociceptive signals in execution of a motor command involving high knee joint dynamics is erroneous. In fact, pain and nociception may leave 


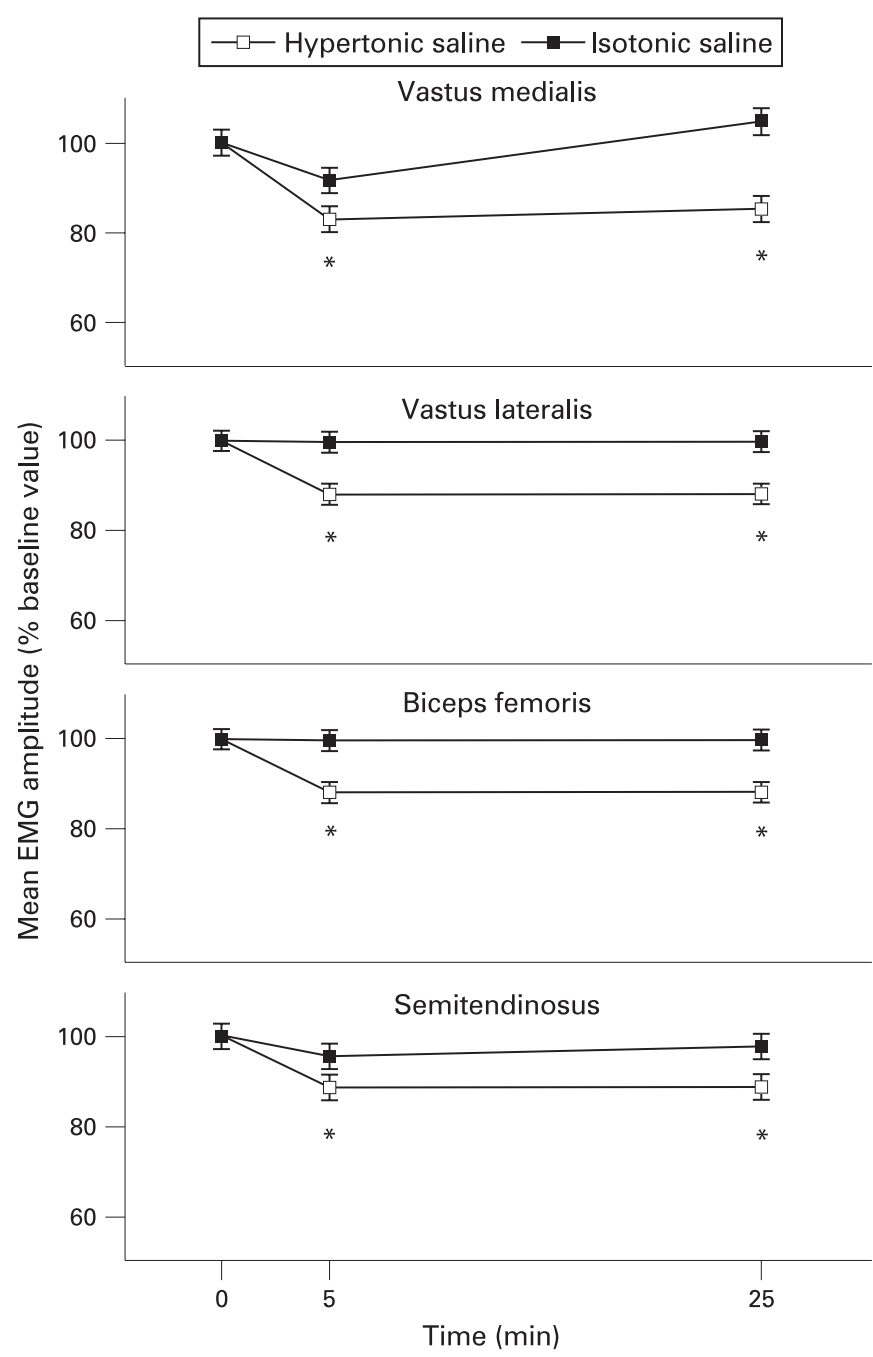

Figure 4 Least-square mean values (SE) of the mean EMG amplitudes, normalised to the Time 0 values, for effects of isotonic (filled symbols) and hypertonic (open symbols) saline injections into the vastus medialis muscle. The injections were given immediately before the measurement at Time 5 minutes. There are statistically significant differences (asterisks) between isotonic and hypertonic injections at Time 5 (during pain) and 25 (after pain) minutes for all muscles.

the knee joint susceptible to injuries during such movements. The findings may have wide implications in the management of muscle pain and prevention of injuries during activities with high knee joint dynamics (e.g. athletic activities).

Acknowledgements: This study was supported by grants from The Oak Foundation, Copenhagen Hospital Corporation and The Danish Physiotherapy Association, to whom the authors are grateful.

Competing interests: None.

\section{REFERENCES}

1. Eriksen J, Jensen MK, Sjogren $P$, et al. Epidemiology of chronic non-malignant pain in Denmark. Pain 2003;106:221-8.

2. Woolf AD, Pfleger B. Burden of major musculoskeletal conditions. Bull World Health Organ 2003;81:646-56.

3. Creamer $\mathbf{P}$, Lethbridge-Cejku $\mathbf{M}$, Hochberg MC. Factors associated with functional impairment in symptomatic knee osteoarthritis. Rheumatology (Oxford) 2000;39:490-6.

4. McAlindon TE, Cooper C, Kirwan JR, et al. Determinants of disability in osteoarthritis of the knee. Ann Rheum Dis 1993;52:258-62.

5. Peers $\mathbf{K H}$, Lysens RJ. Patellar tendinopathy in athletes: current diagnostic and therapeutic recommendations. Sports Med 2005;35:71-87.

6. Dugan SA. Sports-related knee injuries in female athletes: what gives? Am J Phys Med Rehabil 2005;84:122-30.
7. Sonn U. Longitudinal studies of dependence in daily life activities among elderly persons. Scand J Rehabil Med Supp/ 1996;34:1-35.

8. Andriacchi TP, Johnson TS, Hurwitz D, et al. Musculoskeletal dynamics, locomotion and clinical applications. In: Mow VC, Huiskes R, eds. Basic Orthopaedic Biomechanics and Mechano-Biology. 3rd edn. Philadelphia: Lippincott Williams \& Wilkins; 2005: 91-121.

9. Henriksen M, Alkjaer T, Lund $\mathrm{H}$, et al. Experimental quadriceps muscle pain impairs knee joint control during walking. J Appl Physiol 2007;103:132-9.

10. Kaufman KR, Hughes C, Morrey BF, et al. Gait characteristics of patients with knee osteoarthritis. J Biomech 2001;34:907-15.

11. Astephen JL, Deluzio KJ, Caldwell GE, et al. Biomechanical changes at the hip, knee, and ankle joints during gait are associated with knee osteoarthritis severity. $J$ Orthop Res 2008;26:332-41.

12. Rudolph KS, Axe MJ, Buchanan TS, et al. Dynamic stability in the anterior cruciate ligament deficient knee. Knee Surg Sports Traumatol Arthrosc 2001;9:62-71.

13. Alkjaer T, Simonsen EB, Jorgensen $U$, et al. Evaluation of the walking pattern in two types of patients with anterior cruciate ligament deficiency: copers and non-copers. Eur J Appl Physiol 2003;89:301-8.

14. Robon MJ, Perell KL, Fang M, et al. The relationship between ankle plantar flexor muscle moments and knee compressive forces in subjects with and without pain. Clin Biomech 2000;15:522-7.

15. Olmstead TG, Wevers HW, Bryant JT, et al. Effect of muscular activity on valgus/ varus laxity and stiffness of the knee. J Biomech 1986;19:565-77.

16. Wang CJ, Walker PS. Rotatory laxity of the human knee joint. J Bone Joint Surg 1974; 56-A:161-70.

17. Louie JK, Mote CD Jr. Contribution of the musculature to rotatory laxity and torsional stiffness at the knee. J Biomech 1987;20:281-300.

18. Pope MH, Johnson RJ, Brown DW, et al. The role of the musculature in injuries to the medial collateral ligament. J Bone Joint Surg 1979;61-A:398-402.

19. Markolf KL, Graff-Radford A, Amstutz HC. In vivo knee stability. A quantitative assessment using an instrumented clinical testing apparatus. J Bone Joint Surg 1978;60-A:664-74

20. Siegel KL, Kepple TM, Stanhope SJ. Using induced accelerations to understand knee stability during gait of individuals with muscle weakness. Gait Posture 2006;23:435-40.

21. Winter DA. Overall principle of lower limb support during stance phase of gait. J Biomech 1980;13:923-7.

22. Simonsen EB, Dyhre-Poulsen $\mathrm{P}$, Voigt $\mathrm{M}$, et al. Mechanisms contributing to different joint moments observed during human walking. Scand J Med Sci Sports 1997;7:1-13.

23. Graven-Nielsen $\mathbf{T}$, Lund $\mathrm{H}$, Arendt-Nielsen $\mathrm{L}$, et al. Inhibition of maximal voluntary contraction force by experimental muscle pain: a centrally mediated mechanism. Muscle Nerve 2002;26:708-12.

24. Alkjaer T, Simonsen EB, Peter Magnusson SP, et al. Differences in the movement pattern of a forward lunge in two types of anterior cruciate ligament deficient patients: copers and non-copers. Clin Biomech (Bristol, Avon) 2002;17:586-93.

25. Vaughan CL, Davis BL, O'Connor JC. Dynamics of human gait. Champaign, Illinois: Human Kinetics Publishers, 1992.

26. Graven-Nielsen T, Mense S. The peripheral apparatus of muscle pain: evidence from animal and human studies. Clin J Pain 2001:17:2-10.

27. Mense S. Nociception from skeletal muscle in relation to clinical muscle pain. Pain 1993;54:241-89.

28. Schomburg ED, Steffens H, Kniffki KD. Contribution of group III and IV muscle afferents to multisensorial spinal motor control in cats. Neurosci Res 1999;33:195-206.

29. Johansson H, Djupsjobacka M, Sjolander P. Influences on the gamma-muscle spindle system from muscle afferents stimulated by $\mathrm{KCl}$ and lactic acid. Neurosci Res 1993;16:49-57.

30. Wang K, Svensson P, Arendt-Nielsen L. Modulation of exteroceptive suppression periods in human jaw-closing muscles by local and remote experimental muscle pain. Pain 1999;82:253-62.

31. Le Pera D, Graven-Nielsen T, Valeriani M, et al. Inhibition of motor system excitability at cortical and spinal level by tonic muscle pain. Clin Neurophysiol 2001;112:1633-41.

32. Sterling M, Jull G, Wright A. The effect of musculoskeletal pain on motor activity and control. J Pain 2001;2:135-45.

33. Ciubotariu A, Arendt-Nielsen L, Graven-Nielsen T. The influence of muscle pain and fatigue on the activity of synergistic muscles of the leg. Eur J Appl Physiol 2004;91:604-14.

34. Ervilha UF, Farina D, Arendt-Nielsen $\mathrm{L}$, et al. Experimental muscle pain changes motor control strategies in dynamic contractions. Exp Brain Res 2005;164:215-24.

35. Schulte E, Ciubotariu A, Arendt-Nielsen L, et al. Experimental muscle pain increases trapezius muscle activity during sustained isometric contractions of arm muscles. Clin Neurophysiol 2004;115:1767-78.

36. Graven-Nielsen T, Svensson P, Arendt-Nielsen L. Effects of experimental muscle pain on muscle activity and co-ordination during static and dynamic motor function Electroencephalogr Clin Neurophysiol 1997;105:156-64.

37. Polianskis R, Graven-Nielsen T, Arendt-Nielsen L. Spatial and temporal aspects of deep tissue pain assessed by cuff algometry. Pain 2002;100:19-26.

38. Slater $\mathbf{H}$, Arendt-Nielsen L, Wright A, et al. Experimental deep tissue pain in wrist extensors--a model of lateral epicondylalgia. Eur J Pain 2003;7:277-88.

39. Shakespeare DT, Stokes M, Sherman KP, et al. Reflex inhibition of the quadriceps after meniscectomy: lack of association with pain. Clin Physiol 1985;5:137-44.

40. Griffin LY, Albohm MJ, Arendt EA, et al. Understanding and preventing noncontact anterior cruciate ligament injuries: a review of the Hunt Valley II meeting, January 2005. Am J Sports Med 2006;34:1512-32. 